\title{
La participación de las audiencias desde distintos enfoques evaluativos - algunas consideraciones éticas y metodológicas
}

\section{The audience participation since distinctly evaluative focusing - some ethic and methodological considerations}

\author{
Esther Martínez Piñeiro*
}

\begin{abstract}
RESUMO
O impulso resultante da avaliação de programas e políticas públicas nos últimos anos levou a um aumento de práticas de avaliação que, distanciandose da avaliação tradicional, outorgam um papel relevante, e inclusivamente hegemônico, aos vários grupos abrangidos em maior ou menor medida pelo programa e sua avaliação. A dita participação é desejável não só do ponto de vista ético, como também metodológico. Nesta linha, apresentamos uma reflexão em torno da participação das audiências nos estudos da avaliação. Em primeiro lugar, abordamos as considerações metodológicas que levam a potenciar esta participação; a seguir, apresentamos os vários tipos de grupos que são chamados a participar. Detemo-nos depois no papel que esses grupos desempenham no projeto e realização destes estudos e na tomada de decisões posteriores segundo três formas de avaliação pluralistas - a avaliação centrada nos implicados, a avaliação participativa e a avaliação potenciadora. Por último, apresentamos algumas considerações sobre as dificuldades éticas e metodo-lógicas que pode acarretar a dita participação. Palavras-chave: políticas públicas, avaliação, participação.
\end{abstract}

* Doctora en Ciencias de la Educación. Área de Métodos de Investigación y Diagnóstico en Educación. Facultad de Ciencias de la Educación. Universidad de Santiago. mpineiro@usc.es 


\begin{abstract}
The impulse experimented by public programs and policies evaluation during the last years has brought about a remarkable increase of evaluative practices that, in contrast to traditional evaluation, grant the stakeholder groups a relevant, even hegemonic, role. The involvement of these groups in the evaluation is highly desirable, not only from an ethical but also from a methodological point of view. From this perspective, a reflection on the audience participation in evaluative studies is presented. To begin with, methodological considerations supporting this participation are put forward. Next, the more habitually involved groups are described. Thereafter the role played by the aforementioned groups in the evaluation design and implementation and in the subsequent decision-making is analysed from three evaluative pluralist approaches: stakeholder based evaluation, participatory evaluation and empowerment evaluation. Finally, some considerations regarding ethical and methodological difficulties that can arise from the above-mentioned participation are discussed.
\end{abstract}

Key-words: public policy, evaluation, participation.

\title{
Introducción
}

La investigación evaluativa ha experimentado un importante desarrollo en los últimos años. Durante este tiempo se han incrementado los estudios evaluativos de programas sociales de diversa índole y se han publicado numerosos artículos, informes y obras centradas en este tipo de investigación aplicada.

Sin duda en España ha contribuido a este impulso la progresiva implantación de una cultura democrática a partir de los años ochenta; cultura que se ha concretado inicialmente en el desarrollo de sistemas de evaluación de programas y servicios públicos con los que la administración rinde cuentas a la ciudadanía del uso de los recursos colectivos. Sin embargo, estas prácticas evaluativas en las que los ciudadanos son, en todo caso, receptores de los resultados o informantes si pertenecen a algún colectivo directamente implicado en el programa, comienzan a convivir con otras, todavía minoritarias, en las que las audiencias juegan un papel activo en todo el proceso. 
La consideración de criterios democráticos, en consonancia con una conceptualización pluralista de la evaluación, es una tendencia que comienza así a cobrar fuerza por encima de otros enfoques (DE MIGUEL DÍAZ, 2000). En esta línea ofrecemos una reflexión en torno a la participación de estos colectivos en la evaluación de los programas públicos, y el papel otorgado desde distintas propuestas evaluativas. Abordamos tres dimensiones de esta cuestión: en primer lugar, qué consideraciones metodológicas llevan a potenciar la participación de estos grupos, a continuación, qué grupos o colectivos vinculados al programa son habitualmente llamados a colaborar, y, por último, en qué cuestiones o etapas de la evaluación colaboran, atendiendo a tres propuestas donde las audiencias son un elemento central, la evaluación orientada a los implicados, la evaluación participativa y la evaluación potenciadora. ${ }^{1}$ Para finalizar, ofrecemos una reflexión sobre algunas consideraciones y dificultades éticas y metodológicas que puede conllevar la participación de dichos grupos.

\section{La participación de las audiencias: justificación ética y metodológica}

La participación de los grupos o colectivos estrechamente relacionados con los servicios y programas financiados con fondos públicos es en la actualidad un derecho social plenamente aceptado, lo que ha conducido en el marco de una cultura democrática a articular sistemas de representación en la administración y gestión de dichas instituciones. Así también, como ya señalamos, se ha producido la articulación progresiva de sistemas de evaluación en los que los ciudadanos más cercanos al programa adquieren cierta relevancia en el desarrollo de la evaluación.

1 Esta es la traducción que hemos empleado para la corriente evaluativa denominada "empowerment evaluation" dado que su finalidad principal, como explicaremos más adelante, es la potenciación de la autonomía de los individuos. 
La participación de estos grupos - denominados en el ámbito de la investigación evaluativa "stakeholders" - puede ser considerada al menos desde dos dimensiones, ética y metodológica. Desde el punto de vista ético esto permite que el control sobre la evaluación deje de estar exclusivamente en manos de la administración, dotando así de capacidad de decisión a aquellos que en mayor o menor medida están afectados por el programa y su evaluación. Los participantes son así corresponsables de dichos estudios al concederles voz y protagonismo en su realización, haciendo suyo un proceso antes ajeno. Esta colaboración conlleva, por lo tanto, una distribución del poder que inicial-mente estaba en manos del cliente de la evaluación al compartir con estos la información sobre el programa, pero también la responsabilidad.

Tal participación responde así a un derecho democrático que se ha ido haciendo realidad, pero además, posibilita, desde el punto de vista metodológico, potenciar principalmente la utilidad de la evaluación, pero también su viabilidad y propiedad, criterios de calidad de los estudios evaluativos. Dicha colaboración, siguiendo los Estándares de evaluación fijados por el COMITÉ CONJUNTO DE ESTÁNDARES PARA LA EVALUACIÓN EDUCATIVA (1998) mejora la calidad de la evaluación ya que:

- Centra el estudio en aquellas cuestiones de interés no sólo para los responsables del programa, principales clientes, sino también para otros colectivos afectados. De esta manera la evaluación dará respuesta a sus necesidades priorizando y seleccionando la información relevante. La evaluación servirá así a un mayor número de intereses.

- Genera tanto una mayor credibilidad del equipo evaluador, como más confianza y aceptación de éste y de los resultados, cuestiones importantes en las evaluaciones de tipo externo, donde los participantes y responsables del programa en ocasiones son reacios a las evaluaciones realizadas por personas ajenas a la institución.

- Posibilita una mejor comprensión del proceso que es así construido por los participantes, o cuando menos seguido a menor distancia. Esta mayor transparencia del proceso, contribuye así a generar una mayor confianza en los resultados del estudio.

- Incrementa el uso de los resultados de la evaluación, y así su mayor impacto, lo que se concreta en la posterior toma de decisiones con consecuencias para el programa y su entorno (WEISS, 1983). 
Este tipo de evaluación posibilita abrir una vía de solución a muchas de las críticas metodológicas que han recibido prácticas evaluativas. Estas con frecuencia han sido tachadas de irrelevantes y poco realistas (weIss,1983). En el primer caso porque centran el estudio en cuestiones del programa que no son importantes para estas audiencias, lo que a veces es debido a que metodológicamente resulta más sencillo recoger información sobre otros aspectos más accesibles para el evaluador. En el segundo porque suelen utilizar indicadores muy exigentes al no conocer el programa en profundidad. En ambos casos la colaboración de las audiencias en el proceso posibilitará tanto enfocar este hacia cuestiones relevantes como conocer el programa desde la perspectiva de los distintos sectores antes de diseñar el plan de evaluación y acercarse así a la teoría implícita del programa, es decir a las distintas perspectivas de cómo funciona el programa (REYNOLDS; WALBERG, 1990) y a las condiciones en que este se lleva a cabo. En todo caso, estas potencialidades éticas y metodológicas no están exentas de dificultades, como expondremos más adelante.

\section{Los principales implicados en la evaluación}

El término inglés "stakeholder" que puede ser traducido por implicado o afectado, es descrito en el ámbito de la investigación evaluativa como el conjunto de personas cuyas vidas se ven afectadas por el programa y su evaluación (weISs, 1983). GUBA y LINCOLN (1981) lo definen como: "distintos grupos interesados en los resultados de la evaluación, bien porque están directamente afectados por las actividades del programa, bien porque ellos son los responsables en tomar decisiones sobre el programa o sobre programas similares en otros lugares o momentos".

Este término, por lo tanto, da cabida a un potencial número de colectivos y grupos que en mayor o menor medida tienen relación con la intervención evaluada, bien porque contribuyeron de alguna manera a su diseño y puesta en marcha, bien porque sus resultados les afectan directa o indirectamente, o incluso porque tienen relación con otro programa social similar que se realice en dicho momento o que vaya a ser puesto en práctica en un futuro. 
WEISs (1983) ofrece una clasificación de los grupos implicados atendiendo al tipo de información que cada uno de ellos puede demandar al estudio evaluativo. Este autor distingue atendiendo a este criterio cuatro grandes colectivos afectados por el programa y su evaluación:

- Los políticos: en el primer grupo se sitúan a los distintos responsables políticos que tienen relación administrativa con el programa evaluado. Dos son las situaciones más frecuentes: por un lado, la administración puede ser directamente responsable del programa cuando es la encargada de ponerlo en marcha con recursos y personal propio; o, por otro, en muchos casos la administración concede fondos públicos a otras instituciones para la realización del programa, por lo que la administración se ve en la obligación de velar por el buen uso de los recursos públicos utilizados por dichas instituciones y de asegurar la eficacia y efectividad de las acciones realizadas. Este grupo, la administración, es el cliente principal de los estudios evaluativos, dejándolos habitualmente en manos de agencias o grupos de expertos externos.

- Los administradores o gestores del programa: en este grupo pueden incluirse aquellos departamentos, instituciones o profesionales responsables del diseño y la dirección y gestión del programa.

- Los que lo llevan a cabo: este grupo es denominado por weIss (1983) como los prácticos, ya que forman parte de él aquellos que ponen en marcha el proceso de intervención. Potencialmente este grupo puede dar cabida a gran diversidad de profesionales con distinto grado de responsabilidad en el programa: formadores, educadores, profesorado, etc.

- Los clientes del programa y las organizaciones ciudadanas: este cuarto grupo está constituido por los participantes en el programa y por los clientes, pero también por otros colectivos de ciudadanos que mantienen alguna relación con el programa ya que se ven afectados por su impacto. WORTHEN, SANDERS y FITZPATRICK (1997) establecen una clarificadora distinción entre los consumidores primarios y los secundarios, es decir entre los participantes y los beneficiarios directos y aquellos grupos que se ven afectados por lo que les sucede a los directamente beneficiados.

Esta amplia clasificación de los grupos implicados puede ser concretada en cada situación específica dando lugar a una mayor diversidad de 
colectivos diferenciados atendiendo a la complejidad del programa, los posibles grupos beneficiarios de sus resultados directos e indirectos y el conjunto de la ciudadanía que pueda verse afectada por él. En todo caso, habitualmente no todos estos grupos son llamados a participar, debido a limitaciones temporales, metodológicas o presupuestarias o al propio contexto evaluativo.

\section{El papel otorgado a los implicados desde distintas propuestas evaluativas}

Muchos evaluadores han resaltado la importancia que tiene la participación de los afectados en el proceso de evaluación, sin embargo, existen diferencias a la hora de determinar que capacidades se le otorgan a estos grupos y en que etapas o cuestiones de dicho proceso valorativo. Efectivamente es esta una cuestión clave donde claramente se manifiestan las diferencias entre expertos en evaluación.

Tradicionalmente, los colectivos con una relación directa con el programa evaluado han mantenido algún tipo de vínculo con el estudio, aun cuando estos no se llevaran a cabo desde posicionamientos que resaltaran la importancia de esta cuestión. Así, en primer lugar, han sido una fuente fundamental de información y, en segundo lugar, aunque en menor medida, han sido receptores de los resultados de la evaluación.

La primera de estas situaciones es la más habitual, los grupos relacionados directamente con el programa han proporcionado información relevante e insustituible sobre el proceso. En este papel hay que situar fundamentalmente a los participantes, y en menor medida a otros beneficiarios y a los profesionales que lo llevaron a cabo. En el caso del primer grupo, es habitual que los usuarios sean consultados durante la realización del programa y tras su finalización para conocer su valoración de determinados elementos considerados relevantes para los evaluadores. En muchos casos esto responde al criterio de satisfacción del cliente o usuario como un referente clave para valorar la calidad del servicio. 
La recepción de los resultados de la evaluación a alguno de los grupos es otra vía de relación con el proceso valorativo que tiene lugar en algunos casos, si bien, la información suministrada no responde habitualmente a los intereses y preocupaciones del grupo que lo recibe. En ocasiones es una respuesta de agradecimiento por parte del equipo evaluador por haber colaborado como informante en el estudio, y no una respuesta a un derecho del que forma parte de lo evaluado.

Estos papeles, como informantes y como receptores de la información están, sin embargo, lejos de ser el que se le otorgan algunos autores desde propuestas evaluativas pluralistas, pertenecientes a lo que GUBA Y LINCOLN (1986) han denominado de cuarta generación, es decir aquellas en que la evaluación es entendida fundamentalmente como un proceso de negociación entre los afectados, los que mantienen intereses y valoraciones distintas sobre el programa.

Desde los años ochenta distintas propuestas han centrado su interés en la colaboración de los afectados en el proceso de evaluación. Dichas perspectivas se han diferenciado tanto por el papel otorgado e dichos grupos como por los principios evaluativos a los que este responde, aunque entre ellas existen muchas coincidencias. Tres son las principales propuestas en esta línea: la "evaluación centrada en los implicados", "evaluación participativa y colaborativa" y, por último, y de más reciente desarrollo, la llamada "evaluación potenciadora". Estos tres enfoques pueden entenderse como un continuum en un progresivo proceso de participación de las audiencias, y de un menor control del evaluador sobre el proceso (FETTERMAN, 2000), como aparece representado en el cuadro siguiente elaborado a partir del ofrecido por FETTERMAN (2000, p. 113) - original de COUSINS; DONOHUE у вцоOм (1996); en el que también se sitúa la evaluación objetiva, donde, como ya señalamos, los implicados son solamente fuente de consulta.

\section{Evaluación orientada a los implicados}

Esta propuesta entiende la participación de los afectados en la evaluación fundamentalmente como un medio adecuado para potenciar la utilidad de los resultados. Bajo esta perspectiva, no sólo el cliente debe demandar 


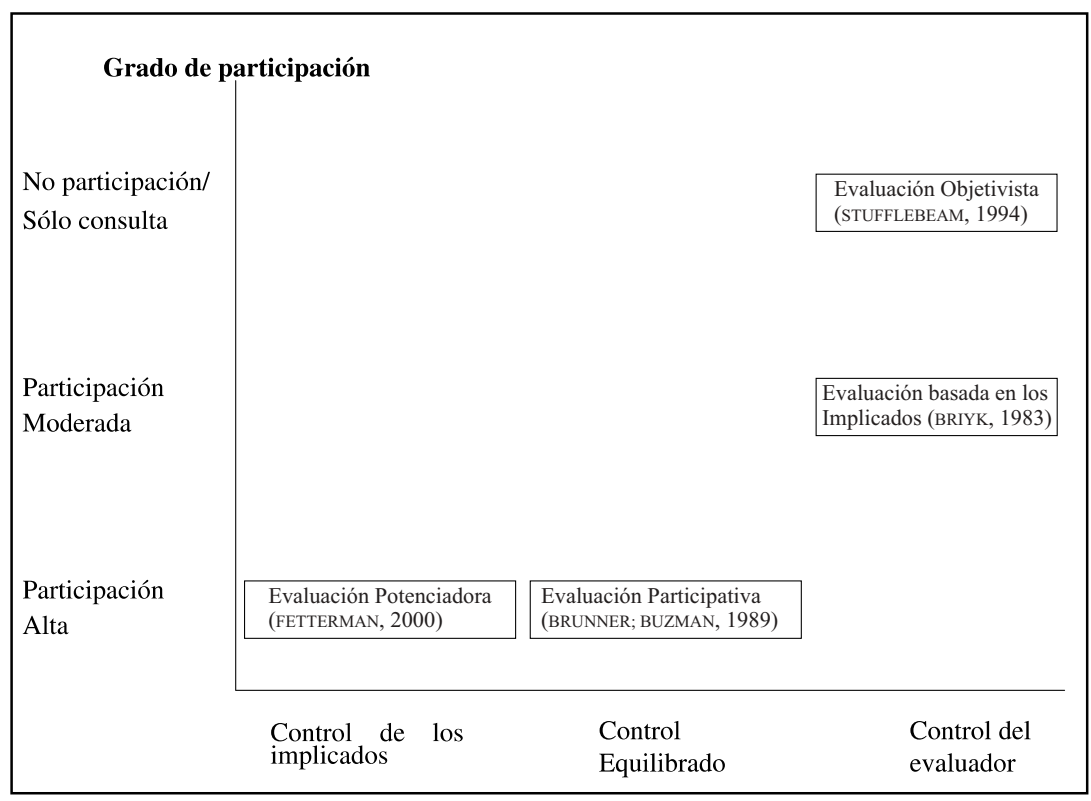

información al evaluador, sino que otros grupos pueden requerir que el estudio ofrezca resultados que propicien la posterior toma de decisiones. wEISS (1983) completa la información sobre las distintas audiencias en la evaluación que antes presentamos exponiendo el tipo de cuestiones que cada uno de ellos habitúa a plantear, y consecuentemente el tipo de resultados de la evaluación que son relevantes para cada uno de ellos. En esta línea, muchos autores (GUBA; LINCOLN, 1981; WEISS, 1983; WORTHEN; SANDERS; FITZPATRICK, 1997) señalan dos ámbitos principales de participación de las audiencias, por un lado, como una referencia a la hora de establecer los aspectos, dimensiones y elementos del programa en los que se debe centrar la evaluación; y, por otro, como fuente de consulta para la selección de criterios de evaluación, cuestiones ambas relevantes en el diseño del proceso.

En el primer caso, el equipo evaluador escucha y recoge información sobre el tipo de información que cada colectivo demanda a la evaluación, 
aquella que les puede resultar útil para hacer planes o elecciones futuras (weIss, 1983). Se pretende de esta manera potenciar la utilidad del proceso al poder así atender las demandas de información de cada uno de estos grupos, aún cuando estas sean diferentes, y no limitarse exclusivamente al cliente de la evaluación. En el segundo caso supone optar por la utilización de criterios descriptivos y democráticos (DE MIGUEL, 2000) -al ceder o compartir así el evaluador la responsabilidad de elección de los criterios con los grupos y personas implicados en el programa.

En la evaluación orientada a los implicados el evaluador desempeña un papel central en el proceso, y en buena manera de él depende el éxito del estudio (GOLD, 1983) ya que debe articular la propuesta evaluativa de forma que puedan tener cabida las demandas e intereses de los distintos grupos; él es así el responsable del diseño de la evaluación y el que toma las distintas decisiones metodológicas.

\section{Evaluación participativa}

Esta corriente evaluativa tiene sus antecedentes en las prácticas de investigación-acción participativa llevadas a cabo a partir de los años setenta en ámbitos tan dispares como la educación de adultos o el desarrollo rural. Es definida por JACKSON y KASSAM (1998) como un proceso de autovaloración, producción de conocimiento colectivo y acción cooperativa en la cual los implicados participan en la identificación de las cuestiones de la evaluación, la recogida y análisis de datos y las acciones tomadas como consecuencia de los resultados de la evaluación. Como puede verse, esta propuesta concede un mayor grado de participación a los grupos relacionados con el programa, ya que estos toman decisiones antes en manos de los evaluadores como la selección de las estrategias de recogida y análisis de información.

La evaluación participativa destaca la función formativa de la evaluación, no ya entendida en su forma más clásica como la que busca el perfeccionamiento y la mejora del programa, sino también como un medio para que los participantes reflexionen sobre su propio trabajo y sean responsables de los cambios que se llevarán a cabo en el programa tras los resultados del estudio. Pretende, por lo tanto desarrollar en los participantes la capacidad reconducir la acción. 
El papel del evaluador bajo este posicionamiento se aleja de su rol tradicional más cercano al de un técnico o investigador. El evaluador participativo debe llegar a acuerdos con los participantes para tomar decisiones conjuntas sobre el desarrollo de la evaluación. Además éste debe formar a personas clave de la organización en cuestiones técnicas y metodológicas para que éstas asuman el papel de coordinadoras del seguimiento y desarrollo del programa; el evaluador será así -llegada esta situación- un consultor experto cuando sea requerido (MERTENS, 1998).

\section{Evaluación potenciadora}

Esta ha sido definida por FetTerman (2000) como el uso de los conceptos, técnicas y resultados de la evaluación para fomentar la autodeterminación. Así, ésta contribuye a que las personas mejoren el programa a través de la autoevaluación y reflexión. La "potenciación" que promueve este tipo de evaluación es entendida como la habilidad de la gente para ganar comprensión y control sobre factores personales, sociales, económicos y políticos para mejorar la situación de sus vidas.

En este contexto la autodeterminación -como habilidad de un sujeto para dirigir el curso de su vida- requiere el desarrollo de diversas capacidades, tales como identificar y expresar necesidades, establecer metas, planificar acciones futuras, hacer elecciones racionales entre alternativas de acción o evaluar resultados a corto y largo plazo.

En la evaluación potenciadora, los implicados conducen el estudio y el evaluador actúa de instructor o de facilitador auxiliar si es preciso (FETTERMAn, 1997). Esta propuesta, como en el caso anterior, mantiene estrechas conexiones con la investigación-acción; sin embargo, en el caso de la evaluación potenciadora, el proceso es conducido por todo el conjunto del grupo, sin que ninguno de los participantes asuma las funciones de dirección o coordinación.

Esta propuesta evaluativa entiende que el enjuiciamiento del mérito o valor del programa no es, como en la evaluación tradicional, el punto final del estudio, sino que aquí comienza un proceso de perfeccionamiento del programa en manos de los grupos implicados. Así también puede entenderse 
como una evaluación de carácter interno, ya que, si bien el evaluador es externo a la institución y el programa, la evaluación es conducida por los propios participantes. Esta propuesta, sin embargo, ha destacado la potencialidad de articular dichos procesos de autoevaluación con sistemas de evaluación externa.

Como se representa en el gráfico anterior, en la evaluación colaborativa y potenciadora, el grado de participación de los implicados es el mismo, pero el control que dichos grupos tienen sobre el proceso evaluador es distinto; en el primer caso implicados y evaluador comparten el control del proceso existiendo una situación de equilibrio; en el segundo, el evaluador deja de tener dicho control cediéndolo a los participantes, de forma que se limita a ser un consultor y son los implicados los que toman todas las decisiones metodológicas sobre el proceso.

Es difícil establecer una línea divisoria entre estas dos propuestas de acción, ya que la participación y colaboración son actividades centrales en ambas. El propio PATTON (1990) entiende que la evaluación participativa es llevada a cabo por los grupos implicados en el programa como un proceso reflexivo para su propio desarrollo y habilitación. FETTERMAN (2000) señala, en un intento de establecer los límites entre ellas, que la evaluación participativa termina cuando los implicados controlan el programa y las decisiones para su mejora, mientras que la evaluación habilitadora comienza en este punto, es decir desde el primer momento son los grupos y sujetos directamente relacionados con el programa los que tienen la capacidad de decidir y de reflexionar sobre su propia práctica y valorarla para cambiar.

La tabla siguiente ofrece las características de cada uno de estos planteamientos en relación a cuatro dimensiones, en primer lugar la finalidad de dicha participación, a continuación el origen de la propuesta, el papel de los implicados y por último el del evaluador. El cuadro se completa con información sobre autores y obras en el marco de cada propuesta evaluativa. 
TABLA 1 - PROPUESTAS EVALUATIVAS Y PAPEL DE LOS IMPLICADOS

\begin{tabular}{|c|c|c|c|}
\hline & $\begin{array}{l}\text { Evaluación basada } \\
\text { en los implicados }\end{array}$ & $\begin{array}{l}\text { Evaluación } \\
\text { participativa }\end{array}$ & $\begin{array}{l}\text { Evaluación } \\
\text { potenciadora }\end{array}$ \\
\hline Finalidad & $\begin{array}{l}\text { Utilidad de los resultados. } \\
\text { Propiciar la toma de } \\
\text { decisiones }\end{array}$ & $\begin{array}{l}\text { Dejar en manos de los } \\
\text { implicados las decisiones } \\
\text { sobre el programa }\end{array}$ & $\begin{array}{l}\text { La autodeterminación: } \\
\text { control del curso de sus } \\
\text { vidas }\end{array}$ \\
\hline Antecedentes & $\begin{array}{llll}\text { Como } & \text { respuesta } & \text { a } & \text { la } \\
\text { escasa } & \text { utilidad } & \text { de } & \text { la } \\
\text { evaluación tradicional } & \end{array}$ & $\begin{array}{l}\text { Investigación-acción } \\
\text { participativa }\end{array}$ & $\begin{array}{l}\text { psicología comunicativa } \\
\text { investigación en acción }\end{array}$ \\
\hline $\begin{array}{l}\text { Papel de los } \\
\text { implicados }\end{array}$ & $\begin{array}{l}\text { Referencia para la } \\
\text { selección de contenidos y } \\
\text { criterios de evaluación }\end{array}$ & $\begin{array}{l}\text { Participa en el diseño y } \\
\text { proceso de evaluación }\end{array}$ & Conduce la evaluación \\
\hline $\begin{array}{l}\text { Papel del } \\
\text { evaluador }\end{array}$ & $\begin{array}{llll}\text { Planifica y } & \text { dirige } & \text { la } \\
\text { evaluación } & & & \\
\end{array}$ & $\begin{array}{lcr}\text { Comparte } & \text { con } & \text { los } \\
\text { implicados } & \text { el } & \text { control } \\
\text { sobre el proceso } & & \end{array}$ & $\begin{array}{l}\text { Es un colaborador y } \\
\text { facilitador del proceso }\end{array}$ \\
\hline Autores & BRIYK (1983) & $\begin{array}{l}\text { BRUNNER y GUZMÁN, } \\
(1989) \\
\text { COUSINS y EARL (1995) }\end{array}$ & FETTERMAN $(1997,2000)$ \\
\hline
\end{tabular}

\section{Dificultades éticas y metodológicas: la otra cara de la moneda}

La elección de un enfoque evaluativo y la aceptación de sus principios más o menos implícitos conlleva también decisiones de tipo metodológico que afectan al planteamiento del estudio y a su realización. De esta forma, optar por un enfoque pluralista y democrático, que promueva la participación de los grupos afectados por el programa supone tener en cuenta algunas cuestiones relativas cuando menos al diseño, la selección de criterios de evaluación, y al propio rol del evaluador.

En primer lugar deberá optarse por un diseño que posibilite la participación de los grupos, es decir flexible y emergente, que permita introducir cambios de acuerdo con las contribuciones de los participantes. Este se flexibiliza cuanto mayor es el grado de participación y de capacidad de decisión sobre el proceso que tengan las audiencias, en un continuo entre los estudios 
en que el evaluador es el responsable de la construcción progresiva del proceso evaluativo articulando las propuestas y demandas de los llamados a participar y aquellas en las que los implicados, como equipo y con el apoyo del evaluador, toman las decisiones sobre el plan y desarrollo de la evaluación. En este continuo, en todo caso, pueden presentarse situaciones complejas, ya destacadas en ocasiones por distintos expertos (WEISS, 1983a; MARK; SHOTLAND, 1987).

Así, el evaluador o las audiencias, deberán conjugar las demandas de los colectivos participantes en la evaluación, pudiendo éstas ser muy diversas, e incluso algunas contrapuestas, lo que desde el punto de vista la operatividad del proceso requiere tomar decisiones sobre cuales serán elemento de evaluación y cuales dejarán de ser atendidas. Esta elección podrá llevarse a cabo teniendo en cuenta la relevancia de la propuesta, el número de personas que la consideren importante, las que se vean afectadas por dicha situación, etc. En todo caso, los responsables de tal elección no podrán evitar tener en cuenta las dificultades metodológicas que puedan existir en la recogida de datos y su análisis por lo que aún cuando no solamente participe el evaluador en decisiones de este tipo, sigue presente el problema real de valorar determinados contenidos o resultados del programa.

Por otra parte, estos procesos evaluativos pueden verse alargados en el tiempo, en el caso de que los grupos participantes en el estudio carezcan de experiencia en situaciones de este tipo donde deben clarificar sus puntos de vista, articularlos con otros dispares, coordinarse, negociar, etc. Esto puede suponer una limitación en la hora de conseguir resultados que puedan repercutir en la mejora del propio proceso, debido a la dificultad de obtenerlos en los plazos de tiempo necesarios para ello.

En conexión con la situación anterior, también se ha señalado la dificultad real de algunos sujetos o grupos invitados a participar para expresar y concretar sus intereses, lo que puede suponer en ocasiones que sus demandas dejen de ser atendidas por falta de claridad y precisión, pero no por tener menor importancia o peso en el estudio. Esta situación puede promover la mayor atención de los intereses de aquellos grupos con una mayor facilidad o experiencia en estas situaciones, los que con frecuencia serán además aquellos grupos que tradicionalmente han contado con una mayor participación en estos estudios.

Bajo estos enfoques pluralistas deja de ser relevante el valor "objetivo" del programa, ya que la atención está puesta en las perspectivas de los distintos grupos y en sus valoraciones y juicios; esta elección de criterios 
democráticos sin la consideración de aquellos de carácter técnico, tradicionalmente aceptados por los expertos en evaluación, también entraña su riesgo, ya que puede quedar sin valorar cuestiones o aspectos técnicamente importantes, pero no considerados por los implicados (MARK; SHOTLAND, 1987). Esta situación puede cuestionar el papel del evaluador en este tipo de estudios y su capacidad para tomar determinadas decisiones metodológicas al tiempo que cabe preguntarse si son realmente relevantes los criterios o cuestiones que pudiendo ser así considerados desde un punto de vista técnico, no lo son para ningún grupo afectado por el programa.

Por último, en las evaluaciones democráticas el rol del evaluador se distancia del tradicional ejercido en estudios realizados desde posicionamientos más clásicos. Este deja de ser fundamentalmente un investigador, un técnico o un especialista para convertirse en un negociador y coordinador. Aparecen nuevas competencias para estos profesionales que requieren una formación no sólo metodológica sino también en conducción y coordinación de grupos, resolución de conflictos, formación de nuevos coordinadores, etc. que les permita realizar adecuadamente estas nuevas funciones.

El diseño y desarrollo de estudios evaluativos bajo planteamientos pluralistas que conlleven la participación de los implicados en el programa, cuando menos en la concreción de contenidos y criterios de avaluación, no está exenta como acabamos de ver, de dificultades metodológicas, pero también de consideraciones éticas a las que debe enfrentarse el equipo evaluador.

Así, la participación de los grupos afectados puede responder, fundamentalmente, a un criterio de utilidad, donde lo que se busca es que el estudio proporcione información relevante para que estos tomen decisiones y elecciones a partir de los resultados. Bajo este punto de vista, el estudio debería potenciar la participación de aquellos que realmente estuvieran en disposición de tomar decisiones sobre el programa y no de todo el conjunto de los colectivos; es decir la evaluación contaría con aquellos que ejercen algun tipo de poder principalmente porque de ellos depende la continuidad del programa, -como la administración-, o porque tengan responsabilidades sobre los profesionales y los recursos, como los gestores y administradores de las instituciones.

Esto supone, por lo tanto, que bajo una posición en principio metodológica, donde se busca la mejora de la calidad del estudio, el evaluador debe tomar una decisión fundamentalmente ética, ya que otorga la palabra a unos colectivos por encima de otros. Esta situación es evitada desde 
posicionamientos más clásicos en los que los criterios y contenidos de la evaluación son decididos por el cliente, en unos casos, o por el propio evaluador desde su posición como experto.

Cuando el criterio de utilidad deja de ser relevante, al menos, en el sentido de consecución de resultados que conlleven cambios en el programa, esta dificultad parece superarse al buscar fundamentalmente la democratización de la evaluación como un proceso de gestión y control de lo público por los propios ciudadanos. Sin embargo, también bajo este prisma la evaluación esconde difíciles decisiones éticas ya que han de articularse no sólo los mecanismos de participación que garanticen la igualdad y la justicia entre los grupos, sino que hay que decidir como se concreta dicha justicia en términos de participación. Cabe preguntarse si todos los grupos deben estar igual de representados, si debe otorgarse mayor espacio e los que menos poder tienen, utilizando así la evaluación como compensadora de una situación social, quien es el que mejor representa a cada grupo e incluso en ocasiones si algunos grupos tienen el derecho mortal a participar (MARK; SHOTLAND, 1987).

Finalmente, existe también el peligro que bajo estos planteamientos evaluativos se esconda únicamente la pretensión de buscar un mayor apoyo social que se concrete en la aceptación del propio estudio y de sus resultados, ya que una evaluación democrática parece moralmente más deseable que aquella que no cuenta con la participación de las audiencias, aun cuando en el fondo no se espere conceder poder real a estos ciudadanos sobre la gestión y el desarrollo de los programas.

\section{Consideraciones finales}

La evaluación democrática supone no sólo un medio para el perfeccionamiento de las instituciones y los servicios que éstas prestan, a través de la valoración de los elementos débiles y fuertes de los programas y de la toma de decisiones conducentes a su mejora, sino que es al mismo tiempo un instrumento para el aprendizaje de la negociación, el acuerdo y la convivencia entre colectivos con objetivos y puntos de vista diferentes. 
Los estudios evaluativos realizados bajo esta perspectiva suponen además, un acto de responsabilidad y compromiso donde los grupos llegan a converger sus diferentes intereses en decisiones comunes. Este tipo de práctica se convierte así en estrategia para el progreso de la cultura democrática y en un signo de madurez institucional, al basar la valoración de los programas públicos no ya exclusivamente en el punto de vista de la administración o del experto, sino en el acuerdo y el consenso de todos los colectivos afectados.

\section{REFERENCIAS}

BRIYK, A. Stakeholder-based evaluation. New Directiones for program evaluation. San Francisco: Jossey Bass, 1983. v. 17.

BROWN, P. The role of the evaluator in Comprehensive Community Initiatives. In: CONNELL, J. P. et al. (Eds.). New approaches to evaluating community initiatives. Washinton: The Aspen Institute, 1995. p. 201-225.

BRUNNER, I.; GUZMAN, A. Participatory evaluation: a tool to assess projets and empower people. In: CONNOR, R. F.; HENDRICKS, M. H. (Eds.). International innovations in evaluation methodology. New Directions for program evaluation. San Francisco: Jossey Bass, 1989. v. 17.

COMITÉ CONJUNTO DE ESTÁNDARES PARA LA EVALUACIÓN EDUCATIVA. Estándares para la evaluación de programas. Bilbao: Mensajero, 1998.

COUSINS, J. B.; EARL, L. M. Paricipatory evaluation in education: studies in evaluation use and organizational learning. London: Falmer Press, 1995.

DE MIGUEL DÍAZ, M. La evaluación de programas sociales: fundamentos teóricos y enfoques metodológicos. Revista de Investigación Educativa, v. 18, n. 2, p. 289-317, 2000.

FETTERMAN, D. M. Empowerment evaluation and accreditation Higher Education. In: CHELIMSKY, E.; SHADIH, W. R. (Eds.). Evaluation for the $21^{\text {st }}$ Century. A Handbook. California: Sage, 1997.

FETTERMAN, D. M. Foundations of empowerment evaluation. California: Sage, 2000.

GOLD, N. Stakeholder and program evaluation: Characterizations and reflextions. In: 
PIÑEIRO, E. M. La participación de las audiencias desde...

BRYK, A. (Ed.). Stakeholder-based evaluation. New directions for program evaluation. San Francisco: Jossey Bass, 1983. v. 17, p. 63-72.

GUBA, E. G.; LINCOLN, Y. S. Effective evaluation. San Francisco: Jossey Bass, 1981.

GUBA, E. G.; LINCOLN, Y. S. The coutenances of fouth-generation evaluation: description, judgment and negotiation. Evaluation Studies Review Annual, v. 11, p 70-88, 1986.

JACKSON, E. T., KASSAM, Y. Knowledge shared: participatory evaluation in development cooperation. West Hartford/Ottawa: Kumarian Press, 1998.

MARK, M. M.; SHOTLAND, R. L. Stakeholder-based evaluation and value judgments. In: CORDRAYS, D. S.; LIPSEY, M. W. (Eds.). Evaluation Studies Review Annual, Beverly Hills, v. 9, n. 5, p. 605-625, 1987.

MERTERNS, D. M. Research methods in Education and Psycology. Thousand Oaks: Sage, 1998.

PATTON, M. Q. Qualitative evaluation methods. Beverly Hills: Sage, 1990.

REYNOLDS, A. J.; WALBERG, H. J. Program theory in evaluation. In: WALBERG, H. J.; HAERTEL, G. (Eds.). The international encyclopedia of educational evaluation. Oxford: Pergamon, 1990.

STUFFLEBEAM, D. L. Empowerment evaluation, objetivist evaluation and evaluation standars: Where the future of evaluation should not go and where it needs to go. Evaluation Practice, v. 15, n. 3, p. 321-338, 1994.

WEISS, C. H. The stakeholder approach to evaluation: origins and promise. In: BRIKS, A. S. (Ed.). Stakeholder-based evaluation. New Directions for program evaluation. San Francisco: Jossey-Bass, 1983. v. 17, p. 3-14.

WEISS, C. H. Towatd the future of stakeholder approaches in evaluation. In: A. S. BRIKS, A. S. (Ed.). Stakeholder-based evaluation. New Directions for program evaluation. San Francisco: Jossey-Bass, 1983. v. 17, p. 83-96.

WORTHEN, B. R.; SANDERS, J. R.; FITZPATRICK, J. L. Program evaluation. Alternative approaches and practical guidelines. New York: Longman, 1997.

Texto recebido em 14 mar. 2002 Texto aprovado em 12 maio 2002 